\title{
Desempenho e conversão alimentar de juvenis de carpa capim (Ctenopharyngodon idella) alimentadas com Azolla filiculoides e ração com baixo teor lipídico
}

\section{Growth and feed utilization by juvenile grass carp (Ctenopharyngodon idella) feed with Azolla filiculoides) with low lipid diet}

\author{
Silvia Maria Guimarães de Souza ${ }^{1 *}$; Diego de Oliveira ${ }^{2}$; Caetano Vaz dos Santos ${ }^{3}$; \\ Maria Emilie Corrêa Gomes ${ }^{3}$; Karina Dufech Esteves ${ }^{3}$
}

\section{Resumo}

\begin{abstract}
A oferta de pescado para o consumo humano dependerá mais da expansão do cultivo de peixes que se alimentam dos primeiros níveis tróficos do que daqueles oriundos da pesca extrativa. Plantas aquáticas usadas em fitotratamento de efluentes de aquacultura vêm sendo referidas como uma alternativa alimentar ao uso exclusivo de rações. A Azolla filiculoides é uma pteridófita que apresenta alto nível protéico. Assim, pesquisas sobre as exigências nutricionais de espécies herbívoras são necessárias. O objetivo principal deste estudo foi avaliar o crescimento e eficiência alimentar de juvenis de carpa capim (Ctenopharyngodon idella) alimentadas com três relações $(\mathrm{R})$ entre ração com baixo teor lipídico e a planta aquática in natura (Azolla filiculoides). O experimento foi conduzido em nove reservatórios com capacidade de $300 \mathrm{~L}$ e três indivíduos por unidade (Peso Inicial $=53,43 \mathrm{~g}$ ). A taxa alimentar diária foi de $2,5 \%$, dividida em duas refeições, constituindo três tratamentos com diferentes relações entre a ração e a Azolla filiculoides na matéria seca ( $\mathrm{R}=\mathrm{Ração} / \mathrm{Azolla}), \mathrm{R}=2,5, \mathrm{R}=5 \mathrm{e} \mathrm{R}=10$. A taxa de crescimento específico ( $\mathrm{TCE}=1,39 \%)$ e o ganho peso diário $(\mathrm{GPD}=1,06 \mathrm{~g} / \mathrm{dia})$ foram melhores na relação Ração / Azolla $\mathrm{R}=5$. Palavras-chave: Peixe herbívoro, Ctenopharyngodon idella, planta aquática, ganho de peso, lipídio
\end{abstract}

\begin{abstract}
The total supply of fish available for human consumption will depend more of the expansion of low trophy level fish farming than of wild-caught fish. Aquatic plants used at treatment of aquaculture wastewater have been showed like an alternative to exclusive diet feed. The aquatic fern, Azolla filiculoides is an example with high level of protein. Consequently, more research on feed requirements of herbivorous fish is required. The aim of this study was to evaluate the growth performance and feed efficiency of juvenile grass carp (Ctenopharyngodon idella) fed with three different diets using low lipid feed and water fern (Azolla filiculoides). The experiment was carried out in nine (9) tanks of $300 \mathrm{~L}$ capacity with three fish per tank (initial body weight $=55,43 \mathrm{~g}$ ). Feeding rate was $2.5 \%$ Fish were fed on two times a day with three treatments $(\mathrm{R}=$ Diet/Azolla on dry weight $) \mathrm{R}=2.5 ; \mathrm{R}=5$ and $\mathrm{R}=10$. The specific growth rate $(\mathrm{SGR}=1.39 \%)$, daily growth gain $(\mathrm{DGG}=1.06 \mathrm{~g} /$ day $)$ and were significantly $(\mathrm{p}<0.05)$ affected by treatment and were highest at $\mathrm{R}=5$.
\end{abstract}

Key words: Grass carp, herbivorous fish, dietary lipids, aquatic plant, Azolla filiculoides, growth

\footnotetext{
1 Professora Doutora do Setor de Aquacultura, Departamento de Zootecnia, Faculdade de Agronomia, Universidade Federal do Rio Grande do Sul, Porto Alegre, Rio Grande do Sul, Brasil. E-mail: silsouza@ufrgs.br.

2 Aluno de Graduação, Agronomia, UFRGS.

3 Aluno de Graduação, Medicina Veterinária, UFRGS.

* Autor para correspondência
} 


\section{Introdução}

A análise de cenários futuros indica que a aquacultura vem ocupando seu espaço, podendo-se afirmar que as fazendas de peixe cada vez mais contribuem para o abastecimento global de pescado. Esta tendência depende de duas condições: a redução do uso de farinha e óleo de peixes na ração e a adoção de políticas públicas e privadas de proteção às áreas hídricas da degradação ambiental. Neste contexto, os peixes planctófagos e herbívoros devem ser priorizados (NAYLOR et al., 2000).

A habilidade dos peixes em utilizar proteína vegetal difere muito, sendo as espécies de hábito alimentar herbívoro, as que melhor a utilizam. No Rio Grande do Sul, a carpa capim (Ctenopharyngodon idella), espécie herbívora originária dos grandes rios da Ásia Central, encontrou um ambiente e um mercado promissores. A carpa capim é utilizada em diversos países, no controle de macrófitas em lagos e canais, sendo observado por Catarino, Ferreira e Moreira (1997) que esta espécie prefere entre várias plantas, a Azolla filiculoides e a Lemna sp. As exigências nutricionais da carpa capim foram estabelecidas por $\mathrm{Du}$ et al. (2005), sendo elas: 20-40 $\mathrm{g} \mathrm{kg}^{-1}$ de lipídios, 350$400 \mathrm{~g} \mathrm{~kg}^{-1}$ de proteínas e a relação energia/proteína de $7,62 \mathrm{~kJ} \mathrm{~g}^{-1}$, evidenciando que a espécie exige baixo conteúdo energético e alto teor protéico na ração.

As plantas aquáticas flutuantes que vêm sendo usadas com relativo êxito no fitotratamento dos efluentes da aquacultura, são: a Eichhornia crassipes (aguapé), a Pistia stratiotes (alface d' água) e a Azolla filiculoides (Azolla). Seus usos na alimentação animal foram avaliados por Henry-Silva e Camargo (2006) que encontraram teor de (PB) $12,45 \%$ e $15,02 \%$ respectivamente, enquanto Aiub (2006) detectou valor de $21,78 \%$ de PB na Azolla filiculoides, sendo ainda, verificada uma duplicação de biomassa a cada 2,3 dias.

Olukunle e Agboola (2005), trabalhando com a substituição do farelo de soja por farinha de lentilha d'água (Lemna sp.) na ração do bagre africano (Clarias gariepinus), não encontraram diferenças entre os níveis de substituição de $25 \%, 50 \%$ e $75 \%$ para a taxa de crescimento específico (TCE), tendo as médias variado entre $1,47 \%$ e $1,52 \%$ que, por sua vez, diferiram significativamente do grupo controle cuja taxa foi de 2,46\%. Assim, diversas pesquisas mostram que o uso exclusivo de macrófita aquática não atende às exigências nutricionais da maioria dos peixes. Dessa forma, seu uso deve ser através da inclusão de sua farinha na ração ou como suplemento alimentar. Poucos são os trabalhos que oferecem em diferentes refeições, a planta aquática verde e a ração peletizada.

Esse experimento foi conduzido para estudar uma ração peletizada, com baixo teor de lipídeo, associada à planta aquática Azolla filiculoides in natura no desempenho de juvenis de carpa capim.

\section{Materiais e Métodos}

O estudo foi conduzido no Setor de Aquacultura do Departamento de Zootecnia, da Faculdade de Agronomia da Universidade Federal do Rio Grande do Sul (UFRGS) com duração de 42 dias. Os 27 peixes foram distribuídos em 9 tanques com capacidade de $300 \mathrm{~L}$, constituindo cada um, uma unidade experimental (UE).

A aeração foi constante e feita através de pedra porosa, acoplada a um compressor de ar central e a temperatura de $28{ }^{\circ} \mathrm{C}$ era mantida por aquecedores e termostatos.

Em cada UE foram colocados 03 peixes com peso médio inicial de $55,43 \mathrm{~g} \pm 13,87$ e comprimento médio de $17,79 \mathrm{~cm} \pm 1,64$.

Foi preparada uma ração à base de farelo de soja (70\%), farelo de arroz desengordurado (25\%), farinha de trigo (2\%) e óleo de soja (3\%). Primeiramente foram misturados os ingredientes secos, seguidos da adição de óleo de soja e de $300 \mathrm{~mL} \mathrm{~kg}^{-1}$ de água. Após a ração ser peletizada, os peletes, com $5 \mathrm{~mm}$ de diâmetro, foram e secos durante $24 \mathrm{~h}$ em estufa a $55^{\circ} \mathrm{C}$ e a seguir armazenados a $-20^{\circ} \mathrm{C}$. A planta aquática foi cultivada em tanques com efluentes do cultivo de peixes, bem eutrofizados. As análises das composições proximais das plantas aquáticas e da ração peletizada estão descritas na Tabela 1. 
Tabela 1. Composição proximal da planta aquática Azolla filiculoides e ração peletizada (\%na Matéria Seca)

\begin{tabular}{lcc}
\hline Alimento & Azolla filiculoides & Ração Peletizada \\
\hline Matéria seca & 100 & 100 \\
Umidade & & \\
Matéria orgânica & 83,73 & 92,34 \\
Proteína Bruta & 21,78 & 38,51 \\
Fibra Bruta & 13,76 & 10,36 \\
Extrato Etéreo & 5,18 & 4,52 \\
Cinzas & 16,27 & 7,66 \\
Extrato Nitrogenado & 43,01 & 38,95 \\
\hline
\end{tabular}

Os valores de oxigênio dissolvido, $\mathrm{pH}$ e temperatura foram monitorados diariamente. Três vezes por semana, as excreções dos peixes eram retiradas por sifonamento, com renovação de $10 \%$ da água dos tanques.

O manejo alimentar foi realizado seis dias por semana, com oferecimento de ração pela manhã, e azolla in natura à tarde. Esta era coletada e pesada depois de retirado o excesso de água da planta. Através da relação $(R)$, onde a ração foi representada por (R) e azolla, representada por (A) com base na matéria seca (MS) de ambos os alimentos, foram estabelecidos os três tratamentos, $(\mathrm{R}=$ Ração / Azolla): $\mathrm{R}=2,5 ; \mathrm{R}=5$ e o tratamento $\mathrm{R}=10$. A taxa diária de alimentação foi $2,5 \%$.

As 04 biometrias realizadas distribuíram-se assim, uma no inicio e as demais a cada 14 dias, sendo a alimentação oferecida ajustada de acordo com o aumento da biomassa.

Os índices de desempenho foram calculados segundo Salhi et al. (2004) em que:
Taxa de Sobrevivência $\operatorname{TS}(\%)=$

$\frac{100-(\text { IndivíduosMortos }- \text { IndivíduosVivos })}{\text { IndivíduosVivos }} \times 100$

Ganho de Peso Diário GPD $(\mathrm{g})=$

$$
\frac{\text { PesoFinal - PesoInicial }}{\text { Número.de.dias }}
$$

Taxa de Crescimento Específico TCE =

$$
\frac{\ln \text { PesoFinal }-\ln \text { PesoInicial }}{\text { Número.de.dias }} \times 100 \text {; }
$$

$\ln =\log$. natural

Conversão Alimentar Aparente CAA =

Dieta..oferecida

Ganho.de.peso 
O delineamento experimental foi o inteiramente casualizado. Foram realizadas Análises de Variância ANOVA para medidas repetidas no tempo, com modelos mistos a 5\% de significância. Quando o teste apresentou diferença significativa foi procedido o teste de comparação múltipla de Tukey-Kramer. As análises foram realizadas utilizando-se o pacote estatístico "SAS 9.1.3 for Windows".

\section{Resultados e Discussão}

A taxa de crescimento específico (TCE) mostrou diferença $(p<0,05)$ entre os tratamentos (Tabela 2$)$. A melhor taxa foi a do $\mathrm{R}=5: 1,39 \pm 0,03$, seguidos de $\mathrm{R}=101,15 \pm 0,09$ e $\mathrm{R}=2,5$ 0,95 $\pm 0,13$ respectivamente, que não apresentaram diferença significativa entre si. Houve interação Tempo*Tratamento, e no Tempo 3 o $\mathrm{R}=5$ foi melhor do que o $\mathrm{R}=10$, não diferindo do $\mathrm{R}=2,5$. Fixando o tratamento $\mathrm{R}=10$, a $\mathrm{TCE}$ foi melhor nos períodos 1 e 2, diferindo significativamente no tempo 3, quando apresentou a pior taxa. Isso pode ser explicado pelo fato das plantas no final do período experimental estarem sem vigor, apresentarem perda de raízes, provavelmente pelo incremento da temperatura e radiação solar naquele período. Coehen et al. (2002) trabalhando com mais de uma espécie do gênero Azolla spp., observaram que estas apresentam aproximadamente $30 \%$ de ácidos graxos polinsaturados (PUFAs). Em certas condições experimentais os níveis de PUFA reduziram, sendo, segundo os autores, um indicativo de redução da fotossíntese, talvez causada, por privação de nutrientes resultante da perda de raízes.

Tabela 2. Peso Inicial Individual (Pi), Peso Final Individual (Pf), Taxa de Crescimento Específico (TCE), Ganho de Peso Diário (GPD) e Conversão Alimentar Aparente (CAA) de juvenis de carpa capim (Ctenopharyngodon idella) após serem alimentados durante 42 dias com diferentes relações $(\mathrm{R})$ de ração e azolla.

\begin{tabular}{cccc}
\hline $\begin{array}{c}\text { Tratamentos } \\
\text { Índices de } \\
\text { Desempenho }\end{array}$ & $\mathrm{R}=2,5$ & $\mathrm{R}=5$ & $\mathrm{R}=10$ \\
\hline Pi (g) & $55,84 \pm 9,66$ & & $43,79 \pm 9,31$ \\
Pf (g) & $86,69 \pm 4,46$ & $53,85 \pm 9,23$ & $74,37 \pm 11,54$ \\
$\operatorname{TCE}^{1}(14$ dias $)$ & $0,95^{\mathrm{a}}$ & $91,71 \pm 14,16$ & $1,39^{\mathrm{a}}$ \\
$\operatorname{TCE}^{1}(28$ dias $)$ & $1,11^{\mathrm{a}}$ & $1,39^{\mathrm{a}}$ & $1,54^{\mathrm{a}}$ \\
$\operatorname{TCE}^{1}(42$ dias $)$ & $0,78^{\mathrm{ab}}$ & $1,54^{\mathrm{a}}$ & $0,52^{\mathrm{b}}$ \\
$\operatorname{TCE}^{1}$ Total & $0,95^{\mathrm{b}} \pm 0,13$ & $1,23^{\mathrm{a}}$ & $1,15^{\mathrm{b}} \pm 0,09$ \\
$\mathrm{GPD}^{2}$ Total & $0,66^{\mathrm{b}} \pm 0,21$ & $1,39^{\mathrm{a}} \pm 0,03$ & $0,79^{\mathrm{ab}} \pm 0,39$ \\
$\mathrm{CAA}^{3}$ Total & $2,69^{\mathrm{a}} \pm 1,00$ & $1,06^{\mathrm{a}} \pm 0,31$ & $4,19^{\mathrm{b}} \pm 2,13$ \\
\hline
\end{tabular}

${ }^{a}$ Valores com letras diferentes em uma mesma linha são estatisticamente diferentes pelo teste de Tukey $(\mathrm{P}<0,05)$.

${ }^{1}$ TCE $\left(\%\right.$ ganho dia ${ }^{-1}=[\ln$ Peso final $-\ln$ Peso inicial / dias $]$ x 100.

${ }^{2} \mathrm{GPD}\left(\right.$ ganho em $\left.\mathrm{g} \mathrm{dia}{ }^{-1}\right)=$ Peso final - Peso inicia $1 / \mathrm{n}$ dias

${ }^{3} \mathrm{CAA}=$ Alimento oferecido/ Ganho de peso. 
Olukunle e Agboola (2005) trabalhando com o bagre africano (C. gariepinus), encontraram taxas de crescimento específico entre 1,47 e $1,52 \%$, portanto próximas à encontrada no tratamento $\mathrm{R}=5$ do presente trabalho, utilizando farinha de lentilha d'água (Lemna sp) em substituição parcial do farelo de soja.

No presente estudo a ração foi elaborada com dois sub-produtos agrícolas, de fácil obtenção no Estado, farelo de arroz e farelo de soja, sendo portanto uma dieta de baixo custo, aproximadamente U\$ 197,97 por tonelada. Nesta linha, Tacon e Silva (1997) citaram que alguns piscicultores do Estado de Andhra Pradesh, na Índia, misturam sub-produtos agrícolas e ofereceram às carpas, existindo alguns que usaram exclusivamente o farelo de arroz desengordurado, em sacos suspensos na água. A base alimentar da carpa capim no Rio Grande do Sul tem sido exclusivamente gramíneas cultivadas como o milheto (Pennisetum sp.), Cerva (2003) e o camerum (Pennisetum purpureum) (SILVA et al., 2006).

Mais recentemente estudos desenvolvidos na China com carpa capim, Du et al.(2005) obtiveram a melhor taxa de crescimento específico (TCE) na ordem de $1,30 \%$ com uma ração contendo $4,23 \%$ na MS de lipídio bruto. Os autores citaram que os níveis de lipídios entre 10 e $12 \%$ na MS resultaram em decréscimo de consumo e interrupção destes tratamentos. A ração usada, no presente trabalho, foi formulada, levando-se em consideração o melhor nível de lipídio, isto é, 4,52\% na MS (Tabela 1). Assim os presentes resultados corroboram com os encontrados pelos autores supracitados. Resultados inferiores para a carpa capim, TCE de 1,07 e 1,17\% foram observados por Cai et al (2005) usando ração controle com zero de substituição da farinha de peixe por farinha de feijão castor, as quais não diferiram daquela com substituição de $40 \%$, que apresentaram uma taxa de crescimento específico de 1,00\%.

Em sistema de policultivo recentemente Silva et al. (2006) oferecendo capim camerum (Pennisetum purpureum) às carpas capim, mais milho e ração para os demais peixes, encontraram TCE mais elevados, variando entre $1,81 \%$ a $2,21 \%$ para aquela espécie.

Para a variável GPD não houve interação entre tratamento e tempo. Procedeu-se então a análise dos fatores independentemente. O GPD de 1,06 ganho em $\mathrm{g}$ /dia observado no tratamento $\mathrm{R}=5$ foi melhor que o encontrado no tratamento $R=2,5$, sendo que tratamento $\mathrm{R}=10$, não apresentou diferença.

Alguns trabalhos onde unicamente plantas forrageiras in natura foram oferecidas às carpas não mostraram resultados satisfatórios. Cerva (2003), trabalhando com juvenis de carpa capim em gaiolas com duas densidades, encontraram uma GPD 0,73g/dia e $0,71 \mathrm{~g} /$ dia, oferecendo $4 \%$ de ração e $10 \%$ de milheto (MV) (Pennisetum sp.) ao dia. Mais recentemente, Camargo et al (2006) obtiveram, com a forragem teossinto (Euchlaena mexicana) (MV) o melhor GPD $=0,15 \mathrm{~g} /$ dia entre as quatro rações estudadas.

Com relação à variável CAA, a interação tempo*tratamento não foi significativa. Quanto aos tratamentos, $\mathrm{R}=5(2,54 \pm 0,48)$ e $\mathrm{R}=2,5(2,69 \pm 1,0)$ não diferiram entre si, mas $\operatorname{sim}$ do $\mathrm{R}=10(4,19 \pm 2,13)$. Valores melhores que no presente estudo, foram encontrados por Cerva (2003) com carpas capim juvenis, isto é $1,70 \mathrm{~g}$ de ração extrusada por $\mathrm{g}$ de peso vivo.

Durante o período experimental, a TS dos peixes foi de $100 \%$.

Os parâmetros médios de qualidade de água durante o período experimental foram: OD: $6.57 \pm$ 0,59; $\mathrm{pH}: 7,33 \pm 0,50$ e temperatura: $27,5 \pm 0,96$, ficando dentro dos níveis adequados para a espécie cultivada.(Du et al., 2005)

\section{Conclusões}

Nas condições em que o experimento foi realizado, é possível afirmar que, em uma associação alimentar entre uma ração e uma planta aquática, considerando a matéria seca (MS), os melhores resultados 
encontrados foram, 5 partes de dieta para 1 parte de Azolla filiculoides.

\section{Agradecimentos}

Agradecemos a Helmut Tessmann Indústria e Comércio de Óleos Vegetais Ltda, pela doação do farelo de arroz desengordurado para a realização desse experimento, e ao professor Doutor Sérgio Luis Vieira do Departamento de Zootecnia da UFRGS, pela colaboração no trabalho.

\section{Referências}

AIUB, J. A. S. Fitotratamento de efluente de aquacultura com Azolla filiculoides. 2006. Dissertação (Mestrado em Zootecnia) - Programa de Pós-Graduação em Zootecnia da Universidade Federal do Rio Grande do Sul, Porto Alegre.

CAI, X.; LUO, L.; XUE, M.; WU, X.; ZHAN, W. Growth performance, body composition and phosphorus availability of juvenile grass carp (Ctenopharyngodon idella) as affected by diet processing and replacement of fishmeal by detoxified castor bean meal. Aquaculture Nutrition, Oxford, v. 11, n. 4, p. 293-299, 2005.

CAMARGO, J. B. J.; RADÜNZ NETO, J.; EMANUELLI, T.; LAZZARI, R.; COSTA, M. L.; LOZEKANN, M. E.; LIMA, R. L.; SCHERER, R.; AUGUSTI, P. R.; PEDRON, F. A.; SANTOS, T. Cultivo de alevinos de carpa capim (Ctenopharyngodon idella) alimentados com ração e forragens cultivadas. Revista Brasileira de Agrociências, Pelotas, v. 12, n. 12, p. 211-215, 2006.

CATARINO, L. F.; FERREIRA, M. T.; MOREIRA, I. S. Preferences of grass carp for macrophytes in Iberian grainage channels. Journal of Aquatic Plant Management, Washington, v. 35, n. 2, p. 79-83, 1997.

CERVA, C. Avaliação do cultivo em gaiolas e do beneficiamento da carpa capim (Ctenopharyngodon idella). 2003. Dissertação (Mestrado em Zootecnia) Programa de Pós-Graduação em Zootecnia da Universidade Federal do Rio Grande do Sul, Porto Alegre.
COEHEN, M. F.; MEZIANE, T.; TSUCHIYA, M.; YAMASAKI, H. Feeding deterrence of Azolla in relation to deoxyanthocyanin and fatty acid composition. Aquatic Botany, Amsterdam, v. 74, n. 2, p. 181-187, 2002.

DU, Z. Y.; LIU, Y. J.; TIAN, L. X.; WANG, J. T.; WANG, Y.; LIANG, G. Y. Effect of dietary lipid level on growth, feed utilization and body composition by juvenile grass carp (Ctenopharyngodon idella). Aquaculture Nutrition, Oxford, v. 11, n. 2, p. 139-146, 2005.

HENRY-SILVA, G. G.; CAMARGO, A. F. M. Composição química de macrófitas aquáticas flutuantes utilizadas no tratamento de efluentes de aquacultura. Planta Daninha, Rio de Janeiro, v. 24, n. 1, p. 21-28, 2006.

NAYLOR, R. L.; GOLDBURG, R. J.; PRIMAVERA, J. H.; KAUTSKY, N.; BEVERIDGE, M. C. M.; CLAY, J.; FOLKE, C.; LUBCHENCO, J.; MOONEY, H.; TROELL, M. Effect of aquaculture on world fish supplies. Nature, London, v. 405, n. 6790, p. 1017-1024, 2000.

OLUKUNLE, O.; AGBOOLA, G. O. Growth performance and nutrient utilization of African Catfish (Clarias gariepinus) fingerlings fed diets with graded inclusion levels of duckweed (Lemna sp.). European Journal of Scientific Research, Morrisville, v. 9, n. 2, p.32-41, 2005.

SALHI, M.; BESSONART, M.; CHEDIAK, G.; BELLAGAMBA, M.; CARNEVIA, D. Growth, feed utilization and body composition of black catfish, Rhamdia quelen, fry fed diets containing different protein and energy levels. Aquaculture, Amsterdam, v. 231, n. 1-4, p. 435-444, 2004.

SILVA, L. B.; BARCELLOS, L. J. G.; QUEVEDO, R.M.; SOUZA, S. M. G.; KREUTZ, L. C.; RITTER, F.; FINCO, J. A.; BEDIN; A. C. Alternative species for traditional carp polyculture in southern South America: Initial growing period. Aquaculture, Amsterdam, v. 255, n. 1-4, p. 417428, 2006.

TACON, A. G. J.; SILVA, S. S. Feed preparation and feed management strategies within semi-intensive fish farming systems in the tropics. Aquaculture, Amsterdam, v. 151, n. 1, p. 379-404, 1997. 